\title{
Liderazgo pedagógico directivo en la educación básica regular: Revisión sistemática
}

\author{
Directive Pedagogical Leadership in Regular Basic Education: A Systematic Review
}

Liderança Pedagógica Diretiva na Educação Básica Regular: Uma Revisão Sistemática

\section{ARTÍCULO ORIGINAL}

\author{
Jorge Silva Huaman \\ https://orcid.org/0000-0003-1850-0065 \\ apusilvah1973@,gmail.com \\ Universidad Cesar Vallejo \\ Lima - Perú
}

Felicitas Quispe Merma

https://orcid.org/0000-0003-3264-660X

apus7aqp@gmail.com

Universidad Cesar Vallejo

Lima - Perú
Jaqueline Huaman Vargas

https://orcid.org/0000-0003-4691-9496

jakirsa@hotmail.com

Universidad San Pedro de Lima

Lima - Perú

Recibido 05 de Febrero 2021 | Arbitrado y aceptado 19 de Abril 2021 | Publicado en 28 Julio 2021

\section{RESUMEN}

La comunidad educativa necesita directores líderes pedagógicos competentes, capaces y comprometidos en su labor profesional, para lograr aprendizajes esperados y calidad educativa, a su vez formar líderes en las aulas que promuevan el cambio en la sociedad. El objetivo del presente es analizar artículos referentes a las competencias directrices del líder pedagógico en la gestión escolar. En cuanto método se hizo la búsqueda documental en relación a la variable de los años 2015 al 2020, en español e inglés en las bases de datos de: Redalyc, Scielo, SLD, Scopus, un total 75 artículos de los 54 seleccionados de Latinoamérica y España. El Resultado que resaltamos es la adopción del estilo de liderazgo transformacional, Coaching y democrático imprescindible en la gestión escolar de las instituciones educativas básicas.

Una de las conclusiones es que el liderazgo pedagógico directivo promueve, adquirir competencias, capacidades, habilidades blandas y duras, manejo de instrumentos de gestión escolar.

Palabras clave: Liderazgo pedagógico, director, gestión escolar, líder transformacional, instrumentos de gestión.

\section{ABSTRACT}

The educational community needs competent, capable and committed pedagogical leaders leaders in their professional work, to achieve expected learning and educational quality, in turn to train leaders in the classrooms that promote change in society. The objective of this present is to analyze articles referring to the directive competences of the pedagogical leader in school management. As a method, the documentary search was carried out in relation to the variable from the years 2015 to 2020, in Spanish and English in the databases of: Redalyc, Scielo, SLD, Scopus, a total of 75 articles of the 54 selected from Latin America and Spain. The Result that we highlight is the adoption of the transformational, Coaching and democratic leadership style essential in the school management of basic educational institutions.

One of the conclusions is that the directive pedagogical leadership promotes, acquiring competences, capacities, soft and hard skills, handling of school management instruments.

Keywords: Pedagogical leadership, director, school management, transformational leader, management instruments.

\section{RESUMO}

A comunidade educacional precisa de lideranças pedagógicas competentes, capazes e comprometidas em seu trabalho profissional, para alcançar a aprendizagem e a qualidade educacional esperadas, por sua vez, para formar lideranças em salas de aula que promovam mudanças na sociedade. O objetivo deste presente é analisar artigos referentes às competências diretivas do líder pedagógico em gestão escolar.

Como método, a busca documental foi realizada em relação à variável dos anos de 2015 a 2020, em espanhol e inglês nas bases de dados: Redalyc, Scielo, SLD, Scopus, num total de 75 artigos dos 54 selecionados do latim América e Espanha. O Resultado que destacamos é a adoção do estilo de liderança transformacional, Coaching e democrático essencial na gestão escolar das instituições de ensino básico. Uma das conclusões é que a liderança pedagógica diretiva promove, adquirindo competências, capacidades, soft e hard skills, o manejo dos instrumentos de gestão escolar.

Palavras chave: Liderança pedagógica, diretor, gestão escolar, líder transformacional, instrumentos de gestão. 


\section{Introducción}

Los directores de las organizaciones educativas básicas son llamados en la actualidad lideres pedagógicos, ellos son los responsables de dirigir las instituciones educativas de manera pertinente y acorde a tendencias actuales de la educación que es lograr aprendizajes esperados en los estudiantes donde el líder pedagógico hace las gestiones necesarias con metas trazadas y objetivos definidos en post de lograr calidad educativa.

Los problemas del Liderazgo Pedagógico en la gestión de los directores, son diversos, dado que una mayoría de directores designados y/o encargados no va más allá de convencer, persuadir, dirigir, con base teórica y experticia, lograr convencer a sus seguidores, para que los docentes, padres de familia trabajen de manera grupal, en equipo para lograr aprendizajes esperados en los estudiantes; sin embargo para lograr estas metas y objetivos el director debe mostrar practica de valores, ser ético y moral, demostrar con ejemplos de trabajo de buen desempeño hacia la comunidad educativa, practicando y/o adoptando un estilo de liderazgo transaccional, Coaching y democrático, Higueras, L., García, I., y Martínez, E. (2018).

Los directores de la educación básica son los lideres pedagógicos, que transmiten ese liderazgo hacia los docentes que tienen el rol de formar líderes en las aulas como una estrategia para obtener aprendizajes esperados en los estudiantes a su cargo, de tal forma que el director transmite ese liderazgo a los docentes a su cargo para poder mejorar de manera integral la escuela llamada instituciones educativas de la educación básica. Muñoz, G., Weintein, J., Sembler, M., y Marfán, J. (2019).

De esta manera los directores denominados líderes pedagógicos, al igual que los Gerentes de las empresas privadas deben desarrollar capacidades, competencias, y desempeños directrices que les ayude a lograr resultados positivos en sus gestiones como líderes pedagógicos. En tal sentido el director o el líder pedagógico desempeñan un papel fundamental en el logro de aprendizajes esperados en los estudiantes de la educación básica regular, ya que gracias a sus aciertos en la gestión lograra que las instituciones educativas mejoren en la calidad educativa. (Vidal y Barriga, 2018).

El director líder pedagógico promueve la buena convivencia, el buen clima escolar en los miembros de la comunidad educativa, incentivar un clima acogedor de 
entendimiento mutuo, sin agresiones físicas, psicológicas ni verbales entre sus miembros que lo integran, para así cumplir metas y objetivos sin perder tiempo en cuestiones de mal trato, ciberbulling, acoso escolar, que tenga que distraer la labor y gestión del director. (Díaz \& Poma, 2016).

Es fundamental que el director líder pedagógico de una institución educativa tenga manejo de habilidades blandas y duras. El tema del manejo emocional positivo ante situaciones adversas fortalece la labor y la gestión del director; así mismo el director debe tener amplio conocimiento y capacidad resolutiva de manera oportuna ante diferentes problemas; el buen conocimiento de sus funciones competenciales hará que fluya los problemas para mejorar la institución educativa por consiguiente mejora de la comunidad, que gracias a la influencia positiva del director y la interacción con sus seguidores logre aprendizajes con los estudiantes (Gutiérrez, 2016).

Por otra parte el director Líder pedagógico de las organizaciones educativas básicas en la actualidad presenta serias deficiencias en cuanto a gestión escolar.

El objetivo del artículo de trabajo es Analizar competencias directrices del líder pedagógico en la gestión escolar para lograr aprendizajes esperados y calidad educativa en las organizaciones educativas básicas; en donde la gestión escolar tenga impacto positivo, y que los directores adopten un estilo de liderazgo pedagógico para formar estudiantes que afronte retos en el contexto familiar y social, situación que obliga a dejar las gestión y liderazgo tradicional por estilos de gerencia y liderazgo moderno. De esta manera el estilo de liderazgo transaccional, Coaching y democrático podría significar una mejora y consolidación del liderazgo en las organizaciones educativas; por lo que responderemos a las incógnitas planteadas a continuación.

RQ1. ¿Cuál es el impacto del director líder pedagógico en gestión escolar en las organizaciones educativas básicas en logros de aprendizaje esperado en estudiantes?

RQ2. ¿Cuáles son las competencias directrices del líder pedagógico en la educación básica para lograr calidad educativa?

RQ3. ¿Qué estilo de liderazgo pedagógico deberían adoptar los directores de las organizaciones educativas básicas? 
RQ4. ¿Qué proponen y piensan los autores a cerca del liderazgo pedagógico en gestión escolar para mejorar el nivel de calidad educativa en los niveles básicos?

Para responder a estas preguntas se hace una revisión sistemática de literatura de diferentes artículos de investigación que refieren sobre liderazgo pedagógico del director, por ello en la primera sección se realiza una breve introducción al trabajo de investigación, dentro de la segunda sección, se caracteriza la metodología de investigación realizada, en la tercera sección se hace el desarrollo teórico sobre liderazgo pedagógico, líder, gestión escolar, competencias directrices y estilos de liderazgo, dentro de la cuarta sección presenta resultados, sección quinta conclusiones, en la última parte se presenta las referencias bibliográficas.

\section{Metodología}

Se hizo la búsqueda documental en relación a la variable de estudio: liderazgo pedagógico directivo en las organizaciones educativas básicas publicadas entre los años 2015 y 2020, en los idiomas español - inglés encontrados en la bases de datos de: Redalyc, Scielo, SLD, Scopus, Academia Edu y google. Se revisaron 75 artículos de investigación de los cuales seleccionamos 54 , de ellos procedentes de los países americanos tales como: México, Colombia, estados Unidos, Argentina, Chile, Ecuador, Venezuela, cañada y España.

La búsqueda de la variable se encontraron a partir de los siguientes términos: "liderazgo pedagógico" en Español, educational leadership en inglés, "director" en español, manager en inglés; y como sinónimos de búsqueda se utilizaron con el nombre de: "business leadership" en inglés, "liderazgo empresarial" en español, "Coaching business" en inglés.

Los criterios de exclusión se tomaron de la siguiente manera: no guardar relación con la variable de investigación, por encontrarse en idioma distinto al inglés y español, por tener más de cinco años de antigüedad en la publicación, además por no tener el rigor científico de artículos indexados en revistas científicas. 
Jorge Silva Huaman, Felicitas Quispe Merma, Jaqueline Huaman Vargas

\begin{tabular}{|l|l|l|l|l|l|}
\hline & Revistas & Año & $\begin{array}{l}\mathrm{N}^{\circ} \text { de } \\
\text { revistas }\end{array}$ & Idioma & Países \\
\hline Incluidas & $\begin{array}{l}\text { Scielo,redalyc, } \\
\text { scopus, } \\
\text { Urosario,Urioja }\end{array}$ & $\begin{array}{l}2015- \\
2020\end{array}$ & 54 & $\begin{array}{l}\text { Español- } \\
\text { ingles }\end{array}$ & $\begin{array}{l}\text { Americanos } \\
\text { y españoles }\end{array}$ \\
\hline excluidas & $\begin{array}{l}\text { Todas las } \\
\text { editadas Antes } \\
\text { del 2014 }\end{array}$ & $\begin{array}{l}\text { Antes del } \\
2014\end{array}$ & 19 & $\begin{array}{l}\text { Que no } \\
\text { corresponden } \\
\text { al Español - } \\
\text { ingles }\end{array}$ & $\begin{array}{l}\text { Que no } \\
\text { corresponden } \\
\text { a } \\
\text { Americanos } \\
\text { y españoles }\end{array}$ \\
\hline
\end{tabular}

Fuente: Elaboración propia

El proceso de búsqueda de la literatura sobre el liderazgo pedagógico directivo, estaba pensado en un protocolo antes de iniciar, vale decir que la técnica de búsqueda estaba definida de forma iterativo para potenciar la calidad del trabajo.

Liderazgo pedagógico directivo en la educación básica regular: una Revisión sistemática de la literatura.

\begin{tabular}{ll}
\hline Planificación & $\begin{array}{l}\text { Protocolo } \\
\text { Criterios de inclusión/exclusión }\end{array}$ \\
\hline Búsqueda & $\begin{array}{l}\text { Palabras claves } \\
\text { Términos Similares }\end{array}$ \\
Selección & $\begin{array}{l}\text { Selección preliminar } \\
\text { Selección específica }\end{array}$ \\
Evaluación de calidad & $\begin{array}{l}\text { Lectura completa } \\
\text { Lectura de resúmenes } \\
\text { Extracción y síntesis }\end{array}$ \\
& $\begin{array}{l}\text { Título y autor } \\
\text { Objetivos de literatura }\end{array}$ \\
\hline
\end{tabular}

Fuente: elaboración propia

Para analizar y revisar los contenidos se categorizo en una tabla de análisis y síntesis, por producción en años ascendente desde el año 2015 hasta el año 2020, por orden año, autor-autores, título de investigación, objetivo y la cantidad de muestra utilizada. 
Jorge Silva Huaman, Felicitas Quispe Merma, Jaqueline Huaman Vargas

\begin{tabular}{|c|c|c|c|}
\hline Año & Autor/autores & Título de investigación & $\begin{array}{l}\text { mues } \\
\text { tra }\end{array}$ \\
\hline \multirow[t]{3}{*}{2015} & Gil'Adí, D. & $\begin{array}{l}\text { "El Líder No Nace, sino Se } \\
\text { Hace" liderazgo una decisión } \\
\text { personal }\end{array}$ & 42 \\
\hline & $\begin{array}{l}\text { Tierno, J., } \\
\text { Barrios, Ch., y } \\
\text { Iranso, P. }\end{array}$ & $\begin{array}{l}\text { Dirección escolar y liderazgo } \\
\text { transformacional: Análisis del } \\
\text { desempeño directivo en centros } \\
\text { educativos de Primaria } \\
\text { Tarragona - España. }\end{array}$ & 56 \\
\hline & $\begin{array}{l}\text { Suceta, L., } \\
\text { Ferreira, D., y } \\
\text { Ternero, I. }\end{array}$ & $\begin{array}{l}\text { Proyecto Educativo, retos y } \\
\text { perspectivas del enfoque } \\
\text { interdisciplinar }\end{array}$ & \\
\hline \multirow[t]{2}{*}{2016} & $\begin{array}{l}\text { Díaz Beter \& } \\
\text { Sime Poma. }\end{array}$ & $\begin{array}{l}\text { Convivencia escolar y una } \\
\text { revisión de estudios de la } \\
\text { educación básica en } \\
\text { Latinoamérica }\end{array}$ & 42 \\
\hline & Gutiérrez, F. & $\begin{array}{l}\text { Método PRINCONSER: } \\
\text { Aplicación de la teoría de } \\
\text { interacción universal en la } \\
\text { dimensión humana. }\end{array}$ & \\
\hline \multirow[t]{4}{*}{2017} & $\begin{array}{l}\text { Ritacco \& } \\
\text { Amores }\end{array}$ & $\begin{array}{l}\text { Dirección de centros educativos } \\
\text { y liderazgo pedagógico: }\end{array}$ & 116 \\
\hline & $\begin{array}{l}\text { Núñez, N., y } \\
\text { Días, D. }\end{array}$ & $\begin{array}{l}\text { Perfil de competencias } \\
\text { gerenciales en directores de } \\
\text { organizaciones educativas. }\end{array}$ & $\begin{array}{l}340 \\
\text { profe } \\
\text { sores }\end{array}$ \\
\hline & Sardón, D & $\begin{array}{l}\text { Liderazgo transformacional y } \\
\text { gestión escolar en } \\
\text { organizaciones educativas } \\
\text { primarias. }\end{array}$ & 76 \\
\hline & $\begin{array}{l}\text { Reyes, D., y } \\
\text { Rojas, R. }\end{array}$ & $\begin{array}{l}\text { Estilo de liderazgo } \\
\text { predominante en organizaciones } \\
\text { sociales dedicadas a la } \\
\text { educación básica }\end{array}$ & 50 \\
\hline \multirow[t]{4}{*}{2018} & Vidal y Barriga & $\begin{array}{l}\text { Gestión liderazgo directivo y } \\
\text { desempeño docente en la I.E. } \\
\text { "Los Ángeles". Universidad de } \\
\text { San Marcos. }\end{array}$ & \\
\hline & $\begin{array}{l}\text { Anchante y } \\
\text { Soriano }\end{array}$ & $\begin{array}{l}\text { Gestión educativa y desempeño } \\
\text { docente }\end{array}$ & 26 \\
\hline & $\begin{array}{l}\text { Salvador y } \\
\text { Sánchez }\end{array}$ & $\begin{array}{l}\text { Liderazgo de directivos y } \\
\text { compromiso organizacional } \\
\text { Docente }\end{array}$ & 71 \\
\hline & $\begin{array}{l}\text { García, I., } \\
\text { Higueras, L., y } \\
\text { Martínez, E. }\end{array}$ & $\begin{array}{l}\text { Inserción de Comunidades } \\
\text { Profesionales de Aprendizaje } \\
\text { por medio del Liderazgo } \\
\text { asignado. }\end{array}$ & 60 \\
\hline
\end{tabular}


Jorge Silva Huaman, Felicitas Quispe Merma, Jaqueline Huaman Vargas

\begin{tabular}{|l|l|l|l|}
\hline 2019 & $\begin{array}{l}\text { Quintanilla, J., y } \\
\text { Bareda, M. }\end{array}$ & $\begin{array}{l}\text { Liderazgo y cultura de } \\
\text { Influencia en la eficacia } \\
\text { organizacional. }\end{array}$ & 32 \\
\cline { 2 - 4 } & $\begin{array}{l}\text { (Fuentes, 2019) } \\
\text { once formas de definir la figura } \\
\text { del líder exponencial }\end{array}$ & 50 \\
& $\begin{array}{l}\text { Suñoz, G., } \\
\text { Mafán, J. }\end{array}$ & $\begin{array}{l}\text { Un decenio de investigación } \\
\text { empírica sobre liderazgo } \\
\text { educacional en Chile. }\end{array}$ & 1200 \\
\hline 2020 & Tilinto, H. & $\begin{array}{l}\text { Estrategias didácticas y } \\
\text { habilidades sociales en la } \\
\text { formación del liderazgo desde la } \\
\text { educación básica. }\end{array}$ & 116 \\
\hline
\end{tabular}

\section{Fuente: elaboración propia}

\section{Desarrollo}

\subsection{Que es el Líder}

Antes de definir pedagogía, directivo y educación básica regular, es necesario definir la palabra líder, liderazgo y otras denominaciones similares, pero con significado relativo y/o diferente.

El Líder No Nace, sino Se Hace, por ello liderazgo es una decisión personal, que pasa por la actitud de hacer bien las cosas por el propio líder, adquirir capacidades y competencias, desarrollar habilidades por iniciativa propia es hacerse un líder de carrera, un líder campo para generar cambios en las organizaciones educativas y empresas donde labora. De ello podemos decir que las características principales para dar identificar un líder por su capacidad de cambiar, innovar, crear, con ideas diferentes y novedosas. El líder en sí, se escoge y selecciona por sus capacidades, talentos, por la forma de persuadir a sus pares, por sus ideales, por accionar positivo, por la práctica constante de valores, por ser ético y moral en sus actos, son cualidades básicas con que debe contar un líder. (Gil’Adí, 2015).

Para Martínez, El Líder imperfecto lo define haciendo algunas preguntas y respuestas, ¿de cómo un líder es capaz de movilizar simpatizantes en grandes masas en post de su propósito?, ¿los lideres nacen o se forman líder?, tomando como punto de partida de que toda conducta es modificable en el campo de la experiencia, podemos mencionar que las cualidades del líder son aprendidas en diferentes situaciones de su 
labor, en diferentes conversaciones, en diferentes capacitaciones, charlas, principalmente realizando estudios de post grado de maestro y Doctorado donde uno se nutre de conocimientos que forman parte de nuestro amplio repertorio. Es necesario reflexionar sobre liderazgo que en el tiempo ha trascendido y ha sido fundamental en la orbe de la empresa, en gestión de organizaciones educativas, donde el Líder Imperfecto se muestra como un espacio para promover debates, formar nuevos líderes, ser reales y objetivos, analizar dogmas, compartir ideas y conocimientos para un mejor desempeño de la persona (2019, p. 182).

\subsection{Que es el liderazgo}

El Liderazgo es aquella acción de influir y persuadir a las personas practicado por el líder, dirigida a través de procesos comunicativos innatas o aprendidas para obtener diversos objetivos y metas trazadas por el mismo líder, por ello el desarrollar ciertas competencias, el desenvolverse con capacidades diferentes fortalece el trabajo directivo, sin embargo estar al frente de personas profesionales requiere aún más habilidades directrices, para el manejo de la diversidad de grupos humanos, con emociones y culturas diferentes, siendo uno de los pilares la gestión de recursos humanos porque dependerá de cuanto se comprometen sus actores y esto dependerá mucho del líder pedagógico que es el director para conseguir los objetivos y metas en mejora institución. (Chiavenato, 1993)

Fuentes, define once formas la figura del líder, primero como la persona influente a través de la comunicación. Segundo, como la persona hábil para unir grupos, y lograr metas y objetivos comunes. Tercero, es el conductor de colectivos que promueve el trabajo en equipo. Cuarto, persona que tiene cualidades y capacidades para guiar grupos de personas. Quinto, persona líder que utiliza todas sus capacidades y competencias para lograr una identidad de grupo. Sexto, persona hábil para convencer personas y lograr objetivos. Séptimo, como un conjunto de habilidades, destrezas para desempeñar un cargo. Octavo, líder que desarrolla competencias a lo largo del ejercicio profesional. Noveno, líder que mejora el desempeño de sus seguidores. Decimo, líder influente en contextos diferentes. Undécimo, líder que despliega su potencial para el logro de matas. (2019, p. 18).

El director de la escuela como líder pedagógico influente en la comunidad educativa, es un profesional que tiene la responsabilidad de conducir al conjunto de personas que forman una parte de una institución y/o empresa de forma satisfactoria en 
la obtención de objetivos y metas trazadas en los instrumentos de gestión institucional. (Freire y Miranda, 2015)

\subsection{Que es el liderazgo pedagógico}

Para Leithwood \& Riehl, el liderazgo pedagógico define como el acto de influenciar a otras personas para obtener metas y objetivos señalados en los documentos de gestión institucional compartidas y socializados en la colectividad educativa de la institución. También se refieren a la capacidad de fomentar el conocimiento intelectual y a la orientación individual como dimensión fundamental de líder pedagógico, donde los líderes pedagógicos asesoran a su equipo de docente, padre de familia y estudiantes al logro de sus metas y objetivos, a la realización de su labor, demostrando respeto y consideración por sus sentimientos y necesidades individuales. La labor empírica del líder ha podido demostrar que estas acciones de apoyo al logro de metas y objetivos del personal a su cargo influyen positivamente en el entusiasmo del personal docente, genera mayor confianza y compromiso en los componentes de la organización educativa. El desarrollo de las competencias personales, así como la capacidad del líder pedagógico subyacente de influir positivamente en sus talentos, potenciales, competencias, capacidades, motivaciones, formas de pensamiento y actitudes de responsabilidad en el trabajo, son talentos y dimensiones esenciales del líder pedagógico y de la concepción moderna de gestión escolar y desarrollo en las organizaciones educativas básicas. (2014, p. 20).

El liderazgo pedagógico directivo es coadyuvar a la obtención de aprendizaje gradual en los estudiantes con la asesoría y autonomía en lo pedagógico de parte del director líder pedagógico, sin embargo, la dirección del líder no implica forzosamente el crecimiento pleno de la magnitud pedagógica. Sino más bien en fomentar la inercia entre compañeros de labor pedagógica, para lograr de manera conjunta metas y objetivos comunes para la mejora de las instituciones educativas de la educación básica (Ritacco \& Amores et ál., 2017)

El director en su condición de líder pedagógico, tiene la gran responsabilidad de elaborar de manera participativa con la comunidad educativa los documentos de gestión escolar y gestión pedagógica como es el Proyecto Educativo, Proyecto Curricular y el Plan Anual. Los instrumentos de gestión en mención orientan a la obtención de metas y 
objetivos por consiguiente trae resultados de aprendizaje en los estudiantes y gradual mejora de la institución educativa. Sardón, D. (2017).

\subsection{Que es el liderazgo pedagógico en la Educación Básica.}

Según el Artículo 36 de la (Ley 28044, 2003), del ministerio de educación Peruano define la Educación Básica Regular, como la categoría educativa que comprende niveles de Inicial, Primaria y Secundaria educativa, dirigida a niños/niñas, púberes y estudiantes de la edad que se promueven oportunamente por el proceso educativo de acuerdo a su desarrollo físico, afectiva y cognitiva, desde los tres años que inician en el nivel inicial, de los seis años hasta los once años en el nivel primario y desde doce años hasta los dieciséis años que comprende el nivel secundario.

\section{Conclusiones}

El liderazgo pedagógico directivo produce un impacto positivo, en los directores.

De las instituciones educativas de la educación básica, porque promueve, adquirir competencias, capacidades, habilidades blandas y duras, manejo de instrumentos de gestión escolar, capacidad de comunicación asertiva, persuasiva, motivadora, tener conocimientos de la gestión de convivencia y clima escolar escolar, para primero ofrecer satisfacción y bienestar de la comunidad educativa y por consiguiente logros de aprendizaje y calidad educativa.

Respondiendo al RQ3. Los directores líderes pedagógicos de las instituciones educativas de la educación básica, deben adoptar un estilo moderno de gestión escolar, que es el líder transformacional, el Coaching y/o el liderazgo democrático, para lograr aprendizajes esperados y calidad educativa. La respuesta al RQ4. Los autores coinciden en señalar que la idoneidad, capacidad y las habilidades es esencial en el ejercicio profesional de director líder pedagógico en los centros educativos de niveles básicos; así mismo los autores determinan que adoptar el estilo de liderazgo transformacional mejora la calidad educativa. 


\section{Referencias bibliográficas}

Anchante y Soriano (2018) Gestión educativa y desempeño docente

http://repositorio.ucv.edu.pe/bitstream/handle/20.500.12692/28743/anchante_cj. pdf? sequence $=1 \&$ isAllowed $=y$

Barrios, Ch., Iranso, P., y Tierno, J. (2015) DIRECCIÓN ESCOLAR y LIDERAZGO: Análisis del desempeño de la figura directiva en centros de Educación Primaria de Tarragona - España. (Tesis Doctoral Universitat Rovira Virgili). Repositorio institucional. https://www.tdx.cat/bitstream/handle/10803/403206/TESI.pdf?sequence=1

Castillero (2019) tipos y clases de variables.

https://psicologiaymente.com/miscelanea/tipos-de-variables

Chiavenato (1993) Coaching empresarial https://www.oocities.org/favm4/gc/liderfzo.htm

Delfino Ferreira, D., Terrero, I., y Suceta, L. (2015) Proyecto Educativo, desafíos y enfoque interdisciplinar. Revista Edu Sol. 15(50). Universidad de Guantánamo Cuba http://www.redalyc.articulo.oa? id=475747191005

Díaz Better \& Sime Poma (2016) Convivencia escolar: una revisión de estudios de la educación básica en Latinoamérica. Revista Virtual Universidad Católica del Norte. http://revistavirtual.ucn.edu.co/index.php/RevistaUCN/article//801/1321

Ferreyra (2015) Proyecto Educativo Desafíos y óptica desde un enfoque interdisciplinar. https://www.redalyc.org/pdf/4757/475747191005.pdf

Frías (2009) Métodos y Diseños de Investigación - elaboración y comprobación de hipótesis. http://www.uv.es/friasnav Universidad de Valencia

Fuentes (2019) Once formas de definir el rol del líder. https://liderexponencial.es/11definiciones-que-describen-la-figuralider/\#: :text=\%E2\%80\%93\%20EL $\% 20 \mathrm{~L} \% \mathrm{C} 3 \% 8 \mathrm{DDER} \% 20 \mathrm{SEG} \% \mathrm{C} 3 \% 9 \mathrm{AN} \%$ 20CHIAVENATO $\% 3 \mathrm{~A} \% 20 \% \mathrm{E} 2 \% 80 \% 9 \mathrm{C}$, uno $\% 20 \mathrm{o} \% 20$ diversos $\% 20$ objetivos $\% 20$ espec $\%$ C3\%ADficos $\%$ E2\%80\%9D. 
García, I., Higueras, L., y Martínez, E. (2018) Inserción de Comunidades Profesionales de Aprendizaje por medio de un Liderazgo repartido.

https://doi.org/10.15366/reice2018.16.2.007

Gil’Adí (2015) Libro “El Líder No Nace, sino Se Hace” liderazgo una decisión personal https://articulo.mercadolibre.com.ar/MLA-801383713-liderazgo-una-decisionpersonal-daniel-gil-adi- JM\#\&gid=1\&pid=1

Gutiérrez (2016) Método PRINCONSER su aplicación en la teoría de la interacción universal y en la dimensión humana. https://cybertesis.unmsm.edu.pe//20.500.12672/2557

Hernández, Fernández y Baptista (2014) Metodología de Investigación booksmedicos.org. https://scholar.google.com.pe/scholar?q=Hern $\% \mathrm{C} 3 \% \mathrm{~A} 1 \mathrm{ndez},+\mathrm{R} .,+\mathrm{Fern} \% \mathrm{C} 3 \% \mathrm{~A}$

Hodges, Moore, Lockee, Trust, \& Bond (2020) Diferencia de enseñanza remota en emergencia y líneas de Aprendizaje.

https://www.google.com/search?q= $\% 2 \mathrm{C}+$ Moore $\% 2 \mathrm{C}+\mathrm{S} . \% 2 \mathrm{C}+$ Lockee $\% 2 \mathrm{C}+\mathrm{B}$. $\% 2 \mathrm{C}+\mathrm{Trust} \% 2 \mathrm{C}+\mathrm{T} .+\mathrm{y}+\mathrm{Bond} \% 2 \mathrm{C}+\mathrm{A} .+(2020) . \& \mathrm{rlz}=1 \mathrm{C} 1 \mathrm{CHBD}$ esPE906PE906 \&oq $=$ Hodges $\% 2 \mathrm{C}+\mathrm{C} . \% 2 \mathrm{C}+$ Moore $\% 2 \mathrm{C}+\mathrm{S} . \% 2 \mathrm{C}+$ Lockee $\% 2 \mathrm{C}+\mathrm{B} . \% 2 \mathrm{C}+$ Trust $\% 2$ $\mathrm{C}+\mathrm{T} .+\mathrm{y}+$ Bond $\% 2 \mathrm{C}+\mathrm{A} .+(2020) .+\& a q \mathrm{~s}=$ chrome..69i57.1247j0j15\&sourceid $=\mathrm{chr}$ ome\&ie $=\mathrm{UTF}-8$.

Horna (2019) Percepción del nivel de liderazgo pedagógico directivo en organizaciones educativas básicas. http://repositorio.ucv.edu.pe/20.500.12692/34856

Jennifer Quintanilla, J., y Barrera, M. (2018) Liderazgo y cultura de eficiencia organizacional. Revista Publicando, 14(2). Universidad de Guayaquil. http://C:/Users/USER/Downloads/1147-Texto\%20del\%20art\%C3\%ADculo$\underline{4455-1-10-20180402 \% 20(2)}$

Marroquín (2014) Confiabilidad - Validez de los Instrumentos de investigación científica. http://www.une.edu.pe/Titulacion/2013/exposicion/SESION-6Confiabilidad $\% 20 \mathrm{y} \% 20$ Validez $\% 20 \mathrm{de} \% 20$ Instrumentos $\% 20 \mathrm{de} \% 20$ investigacio $\underline{\text { n.pdf }}$ 
Murillo (2014) Innovación social en américa latina.

https://scholar.google.com.pe/scholar?q=INVESTIGACION+APLICADA+SEG $\underline{\mathrm{UN}+\mathrm{MURILLO} \& \mathrm{hl}=}$

Nelson (2015). University social responsibility: A fundamental component in the training of future teachers of the Pedagogy career, of the Salesian Polytechnic University. Ney York.

https://www.google.com/search?q=University + social + responsibility $\% 3 \mathrm{~A}+\mathrm{A}+\mathrm{fu}$

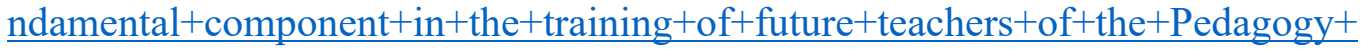
career $\% 2 \mathrm{C}+\mathrm{of}+$ the + Salesian + Polytechnic + University. + Ney + york. $\& r l z=1 \mathrm{ClOK}$ WM enPE899PE899\&oq=University + social + responsibility\%3A+A+fundament $\underline{\text { al }+ \text { component }+ \text { in }+ \text { the }+ \text { training }+ \text { of }+ \text { future }+ \text { teachers }+ \text { of }+ \text { th }}$

Nuguer \& Powell (2020) Informe macroeconómico de América Latina sobre el desempeño de directores. https://doi.org/10.18235/0002284

Núñez, N., Días, D. (2017) Rasgo de competencias gerenciales en directivos de organizaciones educativas básicas.

https://conicyt.scielo.php?script=sci_arttext\&S0718-

Reimers, \& Schleicher (2020) Un marco orientador para una respuesta educativa a la pandemia COVID-19 - 2020. OCDE. https://es.unesco.org/themes/educacionsituaciones-crisis/coronavirus-cierres-escuelas/consecuencias.

Reyes, D., y Rojas, R. (2017) Estilo de liderazgo predominante en organizaciones educativas básicas. Revista Venezolana vol. 22(80). Universidad del Zulia https://www.redalyc.Repo/290/29055967007/html/index.html

Ritacco \& Amores (2017) Dirección escolar y liderazgo pedagógico: un análisis de contenido del discurso de los directores de centros educativos en la Comunidad Autónoma de Andalucía https://www.scielo.br/scielo.php?pid=S1517-es

Salamanca y Crespo (2018) Muestras en la investigación cualitativa. http://www.sc.ehu.es/plwlumuj/praktikak/muestreo 
Salvador y Sánchez (2018) Liderazgo de directivos y compromiso organizacional Docente. Rev. Investig. Altoandin. vol.20 no.1 Puno ene./mar. 2018 http://dx.doi.org/10.18271/ria.2018.335

Sardón, D. (2017) Liderazgo transformacional y la gestión Escolar en organizaciones educativas primarias. Revista de investigación altoandina,19(3), http://www.scielo.org.pe/scielo.php?script=sci arttext\&pid=S2313$\underline{29572017000300007}$

Tolentino, H. (2020) Estrategias didácticas y Habilidades sociales para el desarrollo del liderazgo en la educación básica. Revista innova Educativa. Redalyc: 44(2). Universidad de Costa Rica. https://revistas.ucr.ac.cr/index.php/educacion/article/view/40270/42993

Vidal y Barriga (2018) Gestión liderazgo directivo y desempeño docente en la I.E. "Los Ángeles”. Universidad de San Marcos.

http://igob.edu.pe/ojs/index.php/IGOB/article/view

Weintein, J., Muñoz, G., Senbler, M., y Mafán, J. (2019) Decenio de investigación empírica sobre el liderazgo de centros educativos en Chile. http://doi.org/10.31619/caledu.n51.671 